Check for updates

Cite this: Chem. Sci., 2019, 10, 2404

๑ All publication charges for this article have been paid for by the Royal Society of Chemistry

Received 31st October 2018

Accepted 4th January 2019

DOI: $10.1039 / c 8 s c 04859 b$

rsc.li/chemical-science

\section{Experimental observation of nonadiabatic bifurcation dynamics at resonances in the continuum $\uparrow$}

\author{
Jean Sun Lim, \$ Hyun Sik You, \$§ So-Yeon Kim and Sang Kyu Kim (iD *
}

The surface crossing of bound and unbound electronic states in multidimensional space often gives rise to resonances in the continuum. This situation happens in the $\pi \sigma^{*}$-mediated photodissociation reaction of $2-$ fluorothioanisole; optically-bright bound $\mathrm{S}_{1}\left(\pi \pi^{*}\right)$ vibrational states of 2-fluorothioanisole are strongly coupled to the optically-dark $\mathrm{S}_{2}\left(\pi \sigma^{*}\right)$ state, which is repulsive along the $\mathrm{S}-\mathrm{CH}_{3}$ elongation coordinate. It is revealed here that the reactive flux prepared at such resonances in the continuum bifurcates into two distinct reaction pathways with totally different dynamics in terms of energy disposal and nonadiabatic transition probability. This indicates that the reactive flux in the Franck-Condon region may either undergo nonadiabatic transition funneling through the conical intersection from the upper adiabat, or follow a low-lying adiabatic path, along which multiple dynamic saddle points may be located. Since 2fluorothioanisole adopts a nonplanar geometry in the $S_{1}$ minimum energy, the quasi-degenerate $S_{1} / S_{2}$ crossing seam in the nonplanar geometry, which lies well below the planar $S_{1} / S_{2}$ conical intersection, is likely responsible for the efficient vibronic coupling, especially in the low $S_{1}$ internal energy region. As the excitation energy increases, bound-to-continuum coupling is facilitated with the aid of intramolecular vibrational redistribution, along many degrees of freedom spanning the large structural volume. This leads to the rapid domination of the continuum character of the reactive flux. This work reports direct and robust experimental observations of the nonadiabatic bifurcation dynamics of the reactive flux occurring at resonances in the continuum of polyatomic molecules.

\section{Introduction}

Resonances in the continuum are frequently met in the excitedstate dynamics of photodissociation, ionization, or photodetachment processes. $^{\mathbf{1 - 4}}$ The coupling of discrete and continuum states gives rise to quantum-mechanical interferences when coherently excited states interfere with each other along distinct pathways that lead to an identical final destination. Though a Fano-type resonance has often been reported in atomic systems, it has rarely been observed for polyatomic molecules, for only a few examples including $\mathrm{H}_{3},{ }^{5} \mathrm{FNO}^{6-9}$ or $\mathrm{CH}_{2} \mathrm{~N}_{2} \cdot{ }^{10}$ In some sense, this could be natural since the reaction dynamics in polyatomic systems occur on complicated multidimensional potential energy surfaces. This can hamper the observation of quantum interferences, since so many degrees of freedom are involved in the bound-continuum coupling, and

Department of Chemistry, KAIST, Daejeon 34141, Republic of Korea. E-mail: sangkyukim@kaist.ac.kr

$\dagger$ Electronic supplementary information (ESI) available. See DOI: 10.1039/c8sc04859b

\$ J. S. Lim and H. S. You have contributed equally to this work.

$\S$ Present address: LG Chem, LG science park, Seoul 07796, Republic of Korea.

I Present address: LG Chem, R\&D campus, Daejeon 34122, Republic of Korea. the coherence volume pertaining to a chemical reaction becomes extremely small compared to the entire phase space experienced in resonances in the continuum. But while quantum interferences have rarely been observed, bound-tocontinuum coupling in polyatomic system is ubiquitous in a number of chemical and biological units, and it is believed that such a coupling is essential to the functionalizing of many important nonradiative processes in nature. ${ }^{\mathbf{1 1 , 1 2}}$ In recent decades, one of the very intriguing group of polyatomic molecules in this regard, the heteroaromatic molecular systems such as phenols, ${ }^{13-25}$ thiophenols, ${ }^{26-36}$ or thioanisoles ${ }^{37-46}$ have been subject to intensive and extensive studies. Here, chemical bond dissociation occurs via coupling of bound $\left(\pi \pi^{*}\right)$ to repulsive $\left(\pi \sigma^{*}\right)$ states, which provides great opportunities to investigate nonadiabatic dynamics, conical intersection, or tunnelling at the atomic level. ${ }^{11-46}$ Despite many studies on these systems, however, the detailed mechanism of predissociation on multidimensional potential surfaces in the presence of curve crossings is still beyond our full understanding.

Even though the $\mathrm{S}-\mathrm{CH}_{3}$ bond dissociation of thioanisole occurs on complicated multidimensional potential surfaces, one may describe its reaction pathway using a one-dimensional picture. Namely, the optically bright bound $S_{1}\left({ }^{1} \pi \pi^{*}\right)$ state is crossed by the near-lying dark $S_{2}\left({ }^{1} \pi \sigma^{*}\right)$ state, which is repulsive 
along the $\mathrm{S}-\mathrm{CH}_{3}$ elongation coordinate, Fig. 1 . The $\mathrm{S}_{1} / \mathrm{S}_{2}$ conical intersection seam then acts as a dynamic bottleneck for nonadiabatic transition, through which the reactive flux slides on the repulsive surface to reach the second $S_{0} / S_{2}$ conical intersection located in the later stage of the reaction in planar geometry. The reactive flux at the $\mathrm{S}_{0} / \mathrm{S}_{2}$ conical intersection adiabatically correlates to the $\mathrm{C}_{6} \mathrm{H}_{5} \mathrm{~S} \cdot(\tilde{\mathrm{A}})+\cdot \mathrm{CH}_{3}$ channel, while the lower-lying $\mathrm{C}_{6} \mathrm{H}_{5} \mathrm{~S} \cdot(\tilde{\mathrm{X}})+\cdot \mathrm{CH}_{3}$ fragment channel is opened only when nonadiabatic transition occurs through the $\mathrm{S}_{0} / \mathrm{S}_{2}$ conical intersection. For the thioanisole case, our group has reported a surprising resonant feature in the nonadiabatic transition probability observed at a particular $S_{1}$ vibronic mode. ${ }^{37}$ This mode has been identified through a complete spectroscopic analyses to be the 7a mode corresponding to C-S$\mathrm{CH}_{3}$ asymmetric stretching, which is parallel with the gradient difference vector of the $S_{1} / S_{2}$ conical intersection. As the dissociation along the $\mathrm{S}-\mathrm{CH}_{3}$ elongation coordinate is prompt on the repulsive $S_{2}$ surface, the largely enhanced nonadiabatic transition probability persists at the $\mathrm{S}_{0} / \mathrm{S}_{2}$ conical intersection, favouring the $\mathrm{C}_{6} \mathrm{H}_{5} \mathrm{~S} \cdot(\tilde{\mathrm{X}})+\cdot \mathrm{CH}_{3}$ fragment channel in the asymptotic limit. This thus leads to the resonant-like peak of the $\tilde{\mathrm{X}} / \tilde{\mathrm{A}}$ branching ratio of the $\mathrm{C}_{6} \mathrm{H}_{5} \mathrm{~S}$ - fragment with the $7 \mathrm{a}$ mode excitation of $\mathrm{S}_{1}$ thioanisole.

Further experiments on partially-deuterated thioanisole isotope analogues have demonstrated that vibronic modes showing nonadiabatic dynamic resonances provide a unique way of spectroscopically characterizing the $S_{1} / S_{2}$ conical intersection seam, by means of $S_{1}$ normal modes within the FranckCondon optical window, unless intramolecular vibrational redistribution (IVR) intervenes and dilutes mode-specificity. ${ }^{39,40,42}$ In our recent picosecond time-resolved study, it was revealed that a reactive flux prepared in the proximity of a conical intersection induced by the $7 \mathrm{a}$ mode excitation bifurcates into two distinct reaction pathways. ${ }^{45}$ Remarkably, these two reaction pathways are completely different in terms of their reaction rates, energy disposals, and nonadiabatic

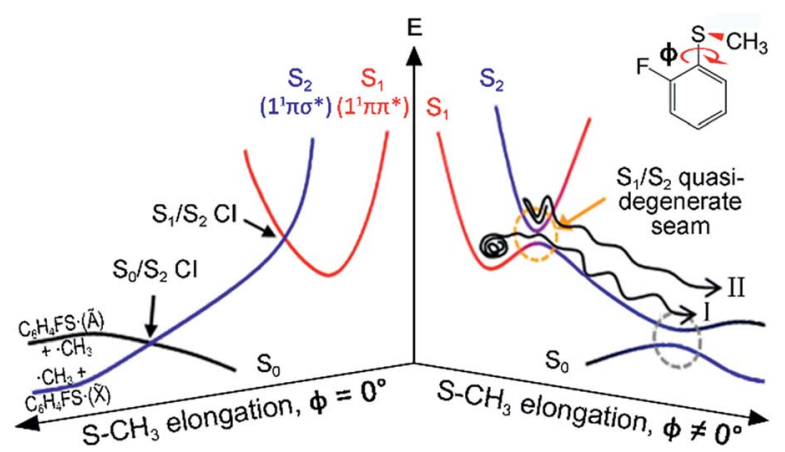

Fig. 1 Schematic of potential energy curves for the photodissociation reaction of 2 -fluorothioanisole. Two conical intersections $(\mathrm{Cls})$ generated at planar geometries become avoided crossings in the nonplanar structure. The dynamics of 2-fluorothioanisole occurs at $\phi$ $\neq 0^{\circ}$ (the right half) due to the nonplanar excited-state geometry. The bifurcated reaction pathways of channel I and II are depicted (see the text). $\phi$ is the $\mathrm{S}-\mathrm{CH}_{3}$ dihedral angle with respect to the plane of benzene moiety. transition probabilities. According to our interpretation, the faster reaction channel with the larger kinetic energy release and larger $\tilde{\mathrm{X}} / \tilde{\mathrm{A}}$ ratio originates from the reactive flux residing in the upper adiabat positioned above the $S_{1} / S_{2}$ conical intersection, through which the nonadiabatic transition to the repulsive $\mathrm{S}_{2}$ surface takes place. Meanwhile, the slower channel with the smaller kinetic energy release and little nonadiabatic transition probability represents the reactive flux seeking the minimum energy path on the low-lying adiabatic potential energy surfaces, leading to the adiabatic product channel of $\mathrm{C}_{6} \mathrm{H}_{5} \mathrm{~S} \cdot(\tilde{\mathrm{A}})+\cdot \mathrm{CH}_{3}$ in the asymptotic limit.

Herein, it is revealed that the nonadiabatic bifurcation dynamics found in the proximity of a conical intersection are generally applicable to the reaction dynamics occurring in the strong bound/unbound coupling regime. This provides a robust explanation for the predissociation dynamics taking place at or near resonances in the continuum of polyatomic molecules. In 2-fluorothioanisole, it is experimentally demonstrated that both adiabatic and nonadiabatic pathways coexist for the reactive flux prepared at resonances in the continuum, and they proceed in a competitive way on multidimensional potential energy surfaces. The nonadiabatic bifurcation dynamics strongly depends on vibronic modes and/or total energy, giving essential information about the multidimensional nature of the nonadiabatic coupling dynamics of polyatomic molecules.

\section{Methods}

\section{Experimental}

2-Fluorothioanisole (TCI, $>98 \%$ ) seeded in Ar carrier gas with a backing pressure of 1-1.5 bar was prepared in a supersonic jet through a pulsed nozzle valve (general valve, $0.5 \mathrm{~mm}$ diameter). The sample was heated to $55{ }^{\circ} \mathrm{C}$ to obtain sufficient vapour pressure. For resonant two-photon ionization (R2PI) spectroscopy, the excitation laser pulse was generated by a frequencydoubled dye laser output (Lambda phisik, Scanmate 2) pumped by a Nd:YAG laser (Surelite II-10). The excitation laser wavelength was scanned from 286 to $270 \mathrm{~nm}$ and crossed with the molecular beam, generating parent ions $\left(\mathrm{C}_{6} \mathrm{H}_{4} \mathrm{FSCH}_{3}{ }^{+}\right)$ when resonant with vibronic transitions. In order to obtain the photofragment excitation (PHOFEX) spectrum, a probe laser pulse was prepared by a tuneable dye laser (Lumonics, HD-500) to ionize the nascent fragment radical $\cdot \mathrm{CH}_{3}(v=0)$ by the $(2+1)$ resonance-enhanced multiphoton ionization at $333.45 \mathrm{~nm}$. The pump laser wavelength was scanned while measuring the $\mathrm{CH}_{3}{ }^{+}$ ion yield. The $\left(1+1^{\prime}\right)$ SEVI spectroscopy ${ }^{47,48}$ was carried out with two independent tuneable dye lasers, Scanmate 2 and Lumonics. The frequency of the pump laser pulse was fixed to a specific vibronic band of $S_{1}$ state. The second laser pulse was used to generate slow photoelectrons from ionization just above the ionization energy threshold. Ionization laser pulses of at least several different wavelengths were employed to get each SEVI spectrum for a specific $S_{1}$ vibronic band. A cloud of photoelectrons was extracted in the low electric field and mapped onto a microchannel plate (MCP) coupled to a phosphor screen. Images were recorded using a charge-coupled device (CCD) camera (Sony XC-ST50), sent to a PC and processed with the 
IMACQ acquisition software. ${ }^{49}$ Photoelectron spectra were obtained by angular integration of images reconstructed via the BASEX algorithm. ${ }^{50}$ The measured electron kinetic energy was plotted according to the internal energy with respect to the band origin of the cationic ground $\left(D_{0}\right)$ state. The VMI method ${ }^{51}$ was used to measure the translational energy and angular distributions of the nascent $\cdot \mathrm{CH}_{3}$ fragment. The wavelength of the pump laser was set to an excitation energy in the range of 286$230 \mathrm{~nm}$. After 10-20 ns, the probe laser with $333.45 \mathrm{~nm}$ was introduced to detect $\cdot \mathrm{CH}_{3}(v=0)$. The polarization of the both pump and probe lasers was perpendicular to the flight direction of the molecular beam and the ion, but parallel to the plane of the MCP and phosphor screen. The anisotropy parameter $(\beta)$ describes the angular distribution of photofragments: $I(\theta)=\sigma /$ $4 \pi\left[1+\beta P_{2}(\cos (\theta))\right]$. Here, $\theta$ is the angle of the fragment velocity vector with respect to the polarization vector (E) of the pump laser, $P_{2}$ is the second-order Legendre polynomial, and $\sigma$ is the absorption cross section.

\section{Computational}

The potential energy curves along $\phi$ (the dihedral angle between the $\mathrm{S}-\mathrm{CH}_{3}$ bond and the ring plane) in the $\mathrm{S}_{0}$ state were calculated using the MP2 (ref. 52) and $\mathrm{B}^{2} \mathrm{LYP}^{53}$ methods with a 6$311++\mathrm{G}(3 \mathrm{df}, 3 \mathrm{pd})$ basis set. The $\phi$ value was varied at $10^{\circ}$ intervals from $0^{\circ}$ to $180^{\circ}$ while the rest of the geometrical parameters were being optimized. A natural bond orbital (NBO) analysis ${ }^{54}$ at local minima (trans and gauche form) in the ground state was performed. The potential energy curves along $\phi$ for the first three singlet excited states were obtained by calculating vertical excitation energies using the equation-of-motion coupled cluster single and doubles (EOM-CCSD) ${ }^{55}$ level of theory at the respective optimized MP2 geometries. Geometries at the conical intersections were optimized using complete active space selfconsistent field (CASSCF) ${ }^{56,57}$ with a $6-311++\mathrm{G}(3 \mathrm{df}, 3 \mathrm{pd})$ basis set. 12 active electrons and 11 active orbitals consist of three pairs of $\pi / \pi^{*}$ orbitals, a nonbonding p orbital of sulfur, $\sigma_{\mathrm{S}-\mathrm{CH}_{3}}$, $\sigma_{\mathrm{S}-\mathrm{CH}_{3}}^{*}, \sigma_{\mathrm{CS}}$, and $\sigma_{\mathrm{CS}}^{*}$ orbitals. The $\mathrm{S}_{1}$ minimum energy was determined by vertical excitation from $\mathrm{S}_{0}$ minimum geometry optimized using the same level of theory. The B3LYP and MP2 calculations were performed using Gaussian 09. ${ }^{58}$ The EOMCCSD and conical intersection optimization calculations were performed using Molpro 2010.1. ${ }^{59}$

\section{Results and discussion}

The reaction dynamics of 2-fluorothioanisole, due to its nonplanar excited-state structure, opens a new aspect of nonadiabatic reaction dynamics by permitting the exploration of the unexplored nuclear configuration space. We carried out spectroscopic characterization of the $S_{1} 2$-fluorothioanisole to confirm its non-planarity, followed by an investigation of stateselective dynamic outputs. This provides deep insights into the nonadiabatic reaction dynamics occurring in the strong coupling regime of bound and unbound states along the nonplanar nuclear coordinates, which are usually inaccessible in systems undergoing optical transition with little structural changes.

In the ground state of 2-fluorothioanisole, the trans-planar structure is predicted to be more stable than gauche by 170 or $150 \mathrm{~cm}^{-1}$ according to B3LYP or MP2 calculations with a basis set of $6-311++\mathrm{G}(3 \mathrm{df}, 3 \mathrm{pd})$, respectively. NBO analysis also supports this, since the delocalization energy of the trans-planar structure is predicted to be $\sim 6.7 \mathrm{kcal} \mathrm{mol}^{-1}$ more stable compared to that of gauche. The preferential population of the trans-planar conformer of 2-fluorothioanisole was also experimentally reported in the solution phase. ${ }^{60}$ We carried out UVUV depletion spectroscopy to confirm that there was only one conformational species in the supersonic jet (see the ESI, Fig. S1 $\dagger$ ), leading us to conclude that the molecular beam of 2fluorothioanisole adopts a trans-planar geometry as the minimum energy structure in the ground state. The R2PI spectrum of 2-fluorothioanisole in the jet (Fig. 2(b)) was found to be quite different from that of thioanisole (Fig. 2(c)) in terms of its overall pattern. Namely, in the case of thioanisole, as the molecule adopts a planar geometry in both the $S_{0}$ and $S_{1}$ states, the $S_{1}-S_{0}$ spectral band origin is the most prominent. ${ }^{37,61-64}$ For 2-fluorothioanisole, however, it was found that the $\mathrm{S}_{1}$ minimum-energy structure becomes nonplanar, as clearly manifested by the strongly observed low vibrational frequency bands at 32 and $82 \mathrm{~cm}^{-1}$ above the $S_{1}$ origin $\left(34974 \mathrm{~cm}^{-1}\right)$. The most probable vibrational mode for these low-frequency bands is the dihedral torsion of the $\mathrm{S}-\mathrm{CH}_{3}$ bond with respect to the plane of the benzene moiety. Eigenvalue calculations using

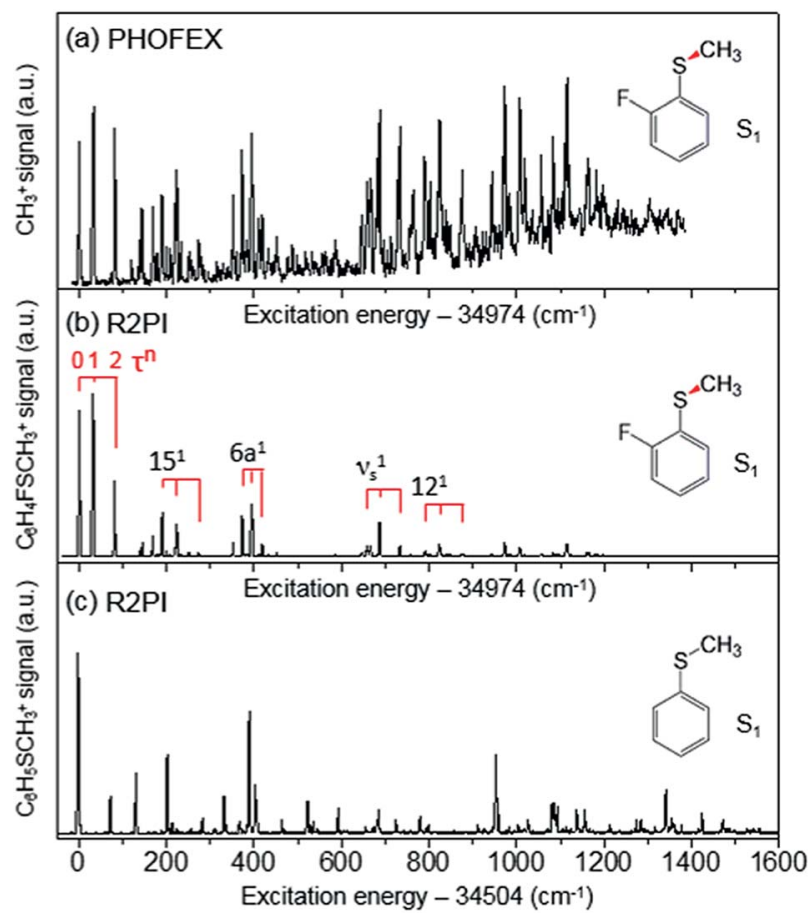

Fig. 2 (a) PHOFEX spectrum of 2-fluorothioanisole taken by monitoring the yield of $\mathrm{CH}_{3}(v=0)$ fragments as a function of the excitation energy. The excitation laser was loosely focused for the PHOFEX spectrum. The R2PI spectra of (b) 2-fluorothioanisole and (c) thioanisole from ref. 37 for comparison. 
a double-well potential energy function of $V(x)=1 / 2 k x^{2}+$ $A \exp \left(-a x^{2}\right)$ reproduced the experimental frequencies and intensities very well when the torsional potential barrier height was $\sim 40 \mathrm{~cm}^{-1}$. Here, $V$ is the potential energy along the $\mathrm{S}^{-} \mathrm{CH}_{3}$ dihedral torsional mode $(x)$ while $k, A$, and a are parameters determining the detailed shape of the double-well potential (see the ESI, Fig. S2 $\dagger$ ). The progression of the $\mathrm{S}-\mathrm{CH}_{3}$ out-of-plane torsional mode was found to be actively combined with other in-plane vibrational modes in the R2PI spectrum, confirming that the largest geometrical change upon the $\mathrm{S}_{1}-\mathrm{S}_{0}$ excitation occurs along the torsional angle of the $\mathrm{S}_{-} \mathrm{CH}_{3}$ bond.

In order to assign $\mathrm{S}_{1}$ vibronic bands and obtain information about IVR, we carried out slow-electron velocity-map imaging (SEVI) spectroscopy, and mode assignments are summarized in Table S1. $\dagger$ According to the propensity rule of $\Delta v=0$ applied when the molecular structure is little changed upon photoionization, it is mostly straightforward to assign many $\mathrm{S}_{1}$ vibronic bands from the $\mathrm{D}_{0}$ modes standing out in the corresponding individual $\left(1+1^{\prime}\right)$ SEVI spectra. It is noteworthy that only the cationic origin band is strongly observed in the SEVI spectra taken via 32 or $82 \mathrm{~cm}^{-1}$, Fig. 3. The same spectral behavior was observed for other molecular systems undergoing large structural change upon ionization..$^{65,66}$ Vibrational bands combined with one quantum of out-of-plane $\tau^{+}$(torsional mode) and $10 \mathrm{~b}^{+}$ modes were persistently observed in the SEVI spectra, indicating that the $\mathrm{D}_{0}-\mathrm{S}_{1}$ transition is accompanied by symmetrybreaking, caused by the nonplanar-to-planar structural change. It is evident that IVR among $\mathrm{S}_{1} / \mathrm{S}_{2}$ vibronic manifolds becomes quite significant at least from the $S_{1}$ internal energy of $658 \mathrm{~cm}^{-1}$, as manifested in the diffuse featureless SEVI spectra taken via $\mathrm{S}_{1}$ intermediate states of the internal energy equal to or higher than $658 \mathrm{~cm}^{-1}$ (Fig. 3). ${ }^{67}$ The IVR dynamics in the $\mathrm{S}_{1} / \mathrm{S}_{2}$ coupling regime is intriguing by itself and provides invaluable information, as it results not only from the density of the $\mathrm{S}_{1}$

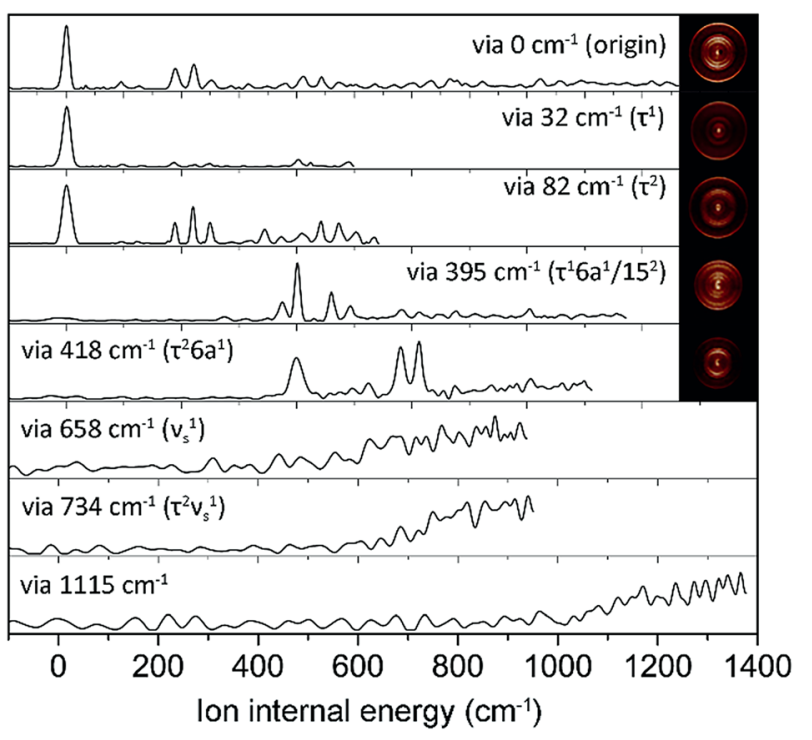

Fig. 3 SEVI spectra of 2-fluorothioanisole via selected $\mathrm{S}_{1}$ intermediate vibronic levels along with assignments in parentheses. Representative photoelectron images are also shown in the inset. vibrational states but also from the $\mathrm{S}_{1} / \mathrm{S}_{2}$ vibronic coupling, which is strongly influenced by the energetic structure of the $\mathrm{S}_{1} /$ $\mathrm{S}_{2}$ conical intersection seam in multidimensional nuclear configurational space. The zero ${ }^{\text {th }}$-order mode character would be no longer guaranteed for vibronic modes observed at $S_{1}$ internal energies higher than $658 \mathrm{~cm}^{-1}$. In SEVI spectrum for the $S_{1}$ internal energy of $418 \mathrm{~cm}^{-1}$, the sign of IVR onset is observed, indicating that the $S_{1} / S_{2}$ coupling already starts to play a major dynamic role in the low internal energy region.

The $a b$ initio calculated structures of 2-fluorothioanisole in the four-lowest singlet electronic states in Fig. 4 are qualitatively consistent with the spectroscopic results. Our EOM-CCSD calculation with a basis set of $6-311++\mathrm{G}(3 \mathrm{df}, 3 \mathrm{pd})$ predicts the $\mathrm{S}_{1}$ minimum structure of the torsional angle of $20^{\circ}$. Even though all of our calculations using TD-DFT, CIS, RICC2, or CASSCF gave the optimized nonplanar $\mathrm{S}_{1}$ structure as the global minimum (Fig. S5 in the ESI $\dagger$ ), the Franck-Condon analysis based on these calculations did not reproduce the intensity pattern in the experiment. The constrained DFT optimization process, freezing $\mathrm{S}$ and $\mathrm{F}$ atoms as well as all carbon atoms of the benzene moiety on the molecular plane, gives rise to the $S_{1}$ minimum structure, which matches the experiment quite well at the torsional angle of $12.5^{\circ}$ (see the ESI, Fig. S3†), even though the corresponding calculated structure gives two imaginary frequencies.

The $\mathrm{S}_{1}$ structural change induced by the simple $\mathrm{H} / \mathrm{F}$ chemical substitution on the ortho-position of thioanisole is expected to have a substantial influence on the nonadiabatic dynamics in the $\mathrm{S}-\mathrm{CH}_{3}$ bond cleavage reaction, since the $\mathrm{S}-\mathrm{CH}_{3}$ dihedral torsional mode is parallel with the coupling vectors of both the $\mathrm{S}_{1} / \mathrm{S}_{2}$ and $\mathrm{S}_{0} / \mathrm{S}_{2}$ conical intersections. ${ }^{38,39}$ The PHOFEX spectrum obtained by monitoring the $\cdot \mathrm{CH}_{3}(v=0)$ fragment yield as a function of the $\mathrm{S}_{1}-\mathrm{S}_{0}$ excitation energy of 2-fluorothioanisole is quite different from the R2PI spectrum. As shown in Fig. 2(a),

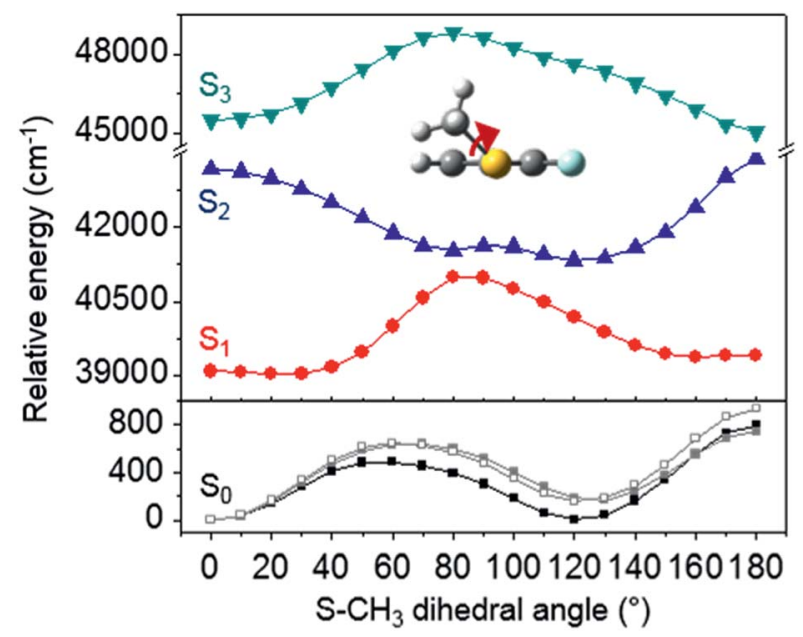

Fig. 4 The four-lowest singlet potential energy curves plotted versus the $\mathrm{S}-\mathrm{CH}_{3}$ dihedral angle of $\mathrm{S}_{0}$ (black), $\mathrm{S}_{1}$ (red), $\mathrm{S}_{2}$ (blue) and $\mathrm{S}_{3}$ (cyan) states. The excited states were calculated using EOM-CCSD/6$311++G(3 d f, 3 p d)$. The relaxed potential energy curves in $S_{0}$ were obtained using the B3LYP (grey filled square) and MP2 (grey empty square) methods with a $6-311++G(3 d f, 3 p d)$ basis set. 
the PHOFEX spectrum shows a continuous background signal that starts to grow from $\sim 400 \pm 100 \mathrm{~cm}^{-1}$ above the $S_{1}$ origin. It gradually increases and persists until the $S_{1}$ internal energy of $\sim 1400 \mathrm{~cm}^{-1}$, indicating that the quantum yield of the $\cdot \mathrm{CH}_{3}(v=$ 0) fragment increases while the R2PI signal diminishes sharply with increasing internal energy. This feature of the PHOFEX spectrum may represent the behaviours of resonances (peaks) in the continuum (background signal) of the $\mathrm{S}_{1}$ 2-fluorothioanisole. The different trends in the R2PI and PHOFEX signals in the high energy region may also result from a decrease in $S_{1}$ lifetime with increasing energy.

Next, the angular and total translational energy distributions of fragments obtained by the VMI method were analysed thoroughly for all of the $S_{1} / S_{2}$ vibronic bands of 2-fluorothioanisole as shown in Fig. 5. The upper limit for the $\mathrm{S}-\mathrm{CH}_{3}$ bond dissociation energy was set to be $67.6 \mathrm{kcal} \mathrm{mol}^{-1}$, which is slightly lower than the 70.8 (ref. 37) (70.5 (ref. 38)) kcal mol ${ }^{-1}$ of thioanisole. At the $S_{1}$ origin, the product translational energy distribution shows a single Gaussian-shaped function peaked at $\sim 17.2 \mathrm{kcal} \mathrm{mol}^{-1}$ although a tiny contribution of the nonadiabatic channel leading to the $\mathrm{C}_{6} \mathrm{H}_{4} \mathrm{FS} \cdot(\tilde{\mathrm{X}})+\cdot \mathrm{CH}_{3}$ fragments is responsible for the slightly asymmetric shape in the high energy region. The $\tilde{\mathrm{X}} / \tilde{\mathrm{A}}$ branching ratio is estimated to be $\sim 0.06$ for 2 fluorothioanisole and this is very similar to that found for thioanisole at the $\mathrm{S}_{1}$ origin. ${ }^{37}$ The obvious bimodal shape of the translational energy distribution is already apparent when the $S_{1}$ internal energy is only $82 \mathrm{~cm}^{-1}$. Even though one may be tempted to attribute this shoulder-like feature in the highenergy region to the one contributed from the nonadiabatic channel giving $\mathrm{C}_{6} \mathrm{H}_{4} \mathrm{FS} \cdot(\tilde{\mathrm{X}})$, this could not be the case simply because the energetic difference between the two deconvoluted Gaussian-shaped distributions $\left(\sim 5 \mathrm{kcal} \mathrm{mol}^{-1}\right)$ is much smaller than the energetic gap between $\mathrm{C}_{6} \mathrm{H}_{4} \mathrm{FS} \cdot(\tilde{\mathrm{A}})$ and $\mathrm{C}_{6} \mathrm{H}_{4} \mathrm{FS} \cdot(\tilde{\mathrm{X}})$ which is $\sim 8.0 \mathrm{kcal} \mathrm{mol}^{-1}\left(2800 \mathrm{~cm}^{-1}\right)$ estimated from the previous studies of 2-fluorothiophenol. ${ }^{31,35}$ This value is slightly smaller than $\sim 8.6 \mathrm{kcal} \mathrm{mol}^{-1}\left(3000 \mathrm{~cm}^{-1}\right)$ measured for that of $\mathrm{C}_{6} \mathrm{H}_{5} \mathrm{~S} \cdot{ }^{68}$ The highest kinetic energy of the fragment is even below the maximum available translational energy of $\mathrm{C}_{6} \mathrm{H}_{4} \mathrm{FS} \cdot(\tilde{\mathrm{A}})$, Fig. 5, and thus this naturally indicates that a new reaction channel (II) mostly giving $\mathrm{C}_{6} \mathrm{H}_{4} \mathrm{FS} \cdot(\tilde{\mathrm{A}})$ with completely different dynamic outputs from those of the existing adiabatic channel (I) starts to be opened (Fig. 1). The growing part of the
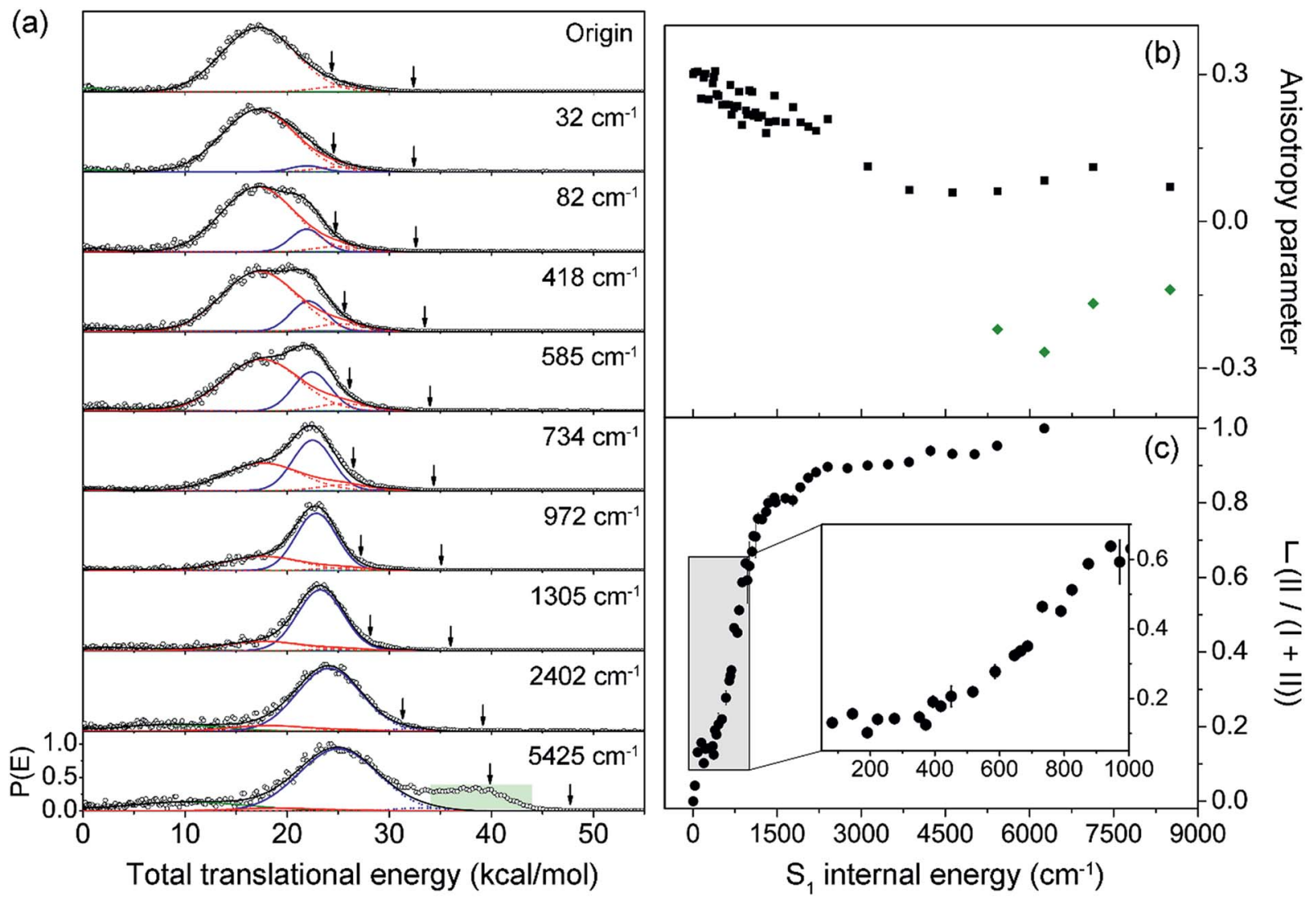

Fig. 5 (a) Total translational energy distributions obtained from velocity-map ion images of $\cdot \mathrm{CH}_{3}$ fragment of 2-fluorothioanisole at pump energies from origin to an $\mathrm{S}_{1}$ internal energy of $5425 \mathrm{~cm}^{-1}$ (black circles). Total distributions are deconvoluted to the low (red lines) and high (blue lines) translational energy channels, both containing $\tilde{A}$ and $\tilde{X}$ states of fragments. Sums of all the fitted lines including minor statistical background most likely originated from multiphoton dissociative ionization (olive lines) are plotted as black lines. The arrows indicate the maximum possible translational energies for channels producing $\tilde{A}$ and $\tilde{X}$ state radicals. (b) Anisotropy parameter $(\beta)$ is plotted as a function of excitation energy (black squares). The $\beta$ values averaged over the range covering the peak at $\sim 40 \mathrm{kcal} \mathrm{mol}^{-1}$ (green shaded area in (a)) are depicted as green diamonds. (c) The fraction $(T)$ of high translational energy channel (II) in the total distribution as a function of the excitation energy (black filled circles). See the ESI† for more details. 
translational energy distribution in the high energy region shows a higher average translational energy than that in the relatively low energy region, meaning that the kinetic energy release in channel II is larger and the internal energy excitation of the fragments is smaller. Reaction channel II is expected to be much faster compared to channel I, leading to narrower distributions of both the internal and translational energies of its fragments, compared to those from I. This is because in channel II there is not enough time for the large volume of phase space to be explored prior to coupling to the repulsive $S_{2}$ state. The anisotropy parameter $(\beta)$ averaged over the entire energy distribution (15 kcal $\mathrm{mol}^{-1}$ ) was found to be slightly positive $(\beta \sim 0.2)$ for all vibronic bands in the $0-2400 \mathrm{~cm}^{-1}$ region above the $S_{1}$ origin as shown in Fig. 5(b). Unfortunately, no information about the transition dipole moments involved in the $S_{1} / S_{2}-S_{0}$ excitation could be inferred from the anisotropy parameters because the excited-state lifetime of 2-fluorothioanisole is not expected to be faster than the rotational period of the entire molecule. ${ }^{45}$

Quite intriguingly, the branching ratio of channel II with respect to channel I increased very rapidly as the excitation energy increased. Firstly, it shows a step-like structure. The yield of channel II $(T \equiv \mathrm{II} /(\mathrm{I}+\mathrm{II}))$ in Fig. 5(c) shows an abrupt increase from 0.04 at $32 \mathrm{~cm}^{-1}$ to 0.13 at $82 \mathrm{~cm}^{-1}\left(\tau^{2}\right)$. And then it remains more or less constant while it fluctuates within the $0.10-0.16$ range up to the excitation energy of $400 \mathrm{~cm}^{-1}$. Incidentally, $\Gamma$ shows slight drops at the 191 and $373 \mathrm{~cm}^{-1}$ bands, associated with the 15 and 6a in-plane vibrational modes, respectively. This in turn indicates that $\Gamma$ increases when out-ofplane modes are involved in the excited state. Thereafter, $\Gamma$ increases dramatically to $\sim 0.8$ at $\sim 1200 \mathrm{~cm}^{-1}$. The dominance of channel II with the increase in excitation energy in this energy region could be attributed to an increase of the accessibility of corresponding quantum states to the upper-lying planar conical intersection, probably because this region includes $\tau$ progressions combined with symmetric and/or asymmetric $\mathrm{C}-\mathrm{S}-\mathrm{CH}_{3}$ stretching modes $\left(v_{\mathrm{s}}\right.$ and/or 7a, respectively) which are parallel to the gradient vector of the conical intersection (Table 1). This could be reflected in the slight but evident enhancement of $\Gamma$ at $\sim 734 \mathrm{~cm}^{-1}$, Fig. 5(c), which is tentatively assigned to $\tau^{2} v_{\mathrm{s}}$. The low translational energy component due to channel I almost completely disappears above $\sim 2400 \mathrm{~cm}^{-1}$. When the excitation energy is higher than $4600 \mathrm{~cm}^{-1}$ above the $S_{1}$ origin, another electronic transition $\left(S_{3}-\right.$ $\mathrm{S}_{0}$ ) comes in and gives rise to a significant contribution of the nonadiabatic channel, giving away the $\mathrm{C}_{6} \mathrm{H}_{4} \mathrm{FS} \cdot(\tilde{\mathrm{X}})$ fragment. The reaction rates at such high energies should be faster than the rotational period of the whole molecule, showing a negative anisotropy parameter of $\sim-0.2$. The dynamics at such high excitation energies are expected to be quite different and subject to further investigation.

The experimental fact that two distinct reaction channels exist, giving completely separable product state distributions indicates that the reactive fluxes prepared at resonances in the continuum prepared in the strong coupling regime of the bound $\left(\mathrm{S}_{1}\right)$ and unbound $\left(\mathrm{S}_{2}\right)$ states of 2-fluorothioanisole bifurcate into two totally different reaction pathways, as in
Fig. 1. Our recent time-resolved work on thioanisole clearly demonstrated that a reactive flux prepared in the proximity of the conical intersection bifurcates into either an adiabatic or nonadiabatic reaction pathway with distinct reaction times, as well as different energy disposals and nonadiabatic transition probabilities. ${ }^{45}$ The nonadiabatic process was measured to be much faster than the adiabatic pathway. That situation is very similar to the one here with 2-fluorothioanisole, except that both the adiabatic and nonadiabatic parts of the initiallyprepared reactive flux in the Franck-Condon region mostly end up with the adiabatic channel $\left(\mathrm{C}_{6} \mathrm{H}_{4} \mathrm{FS} \cdot(\tilde{\mathrm{A}})\right)$ in the asymptotic limit. This is because 2 -fluorothioanisole adopts a nonplanar geometry in both $S_{1}$ and $S_{2}$ as shown in Fig. 4. This theoretical prediction strongly suggests that the reactive flux riding on the repulsive $\mathrm{S}_{2}$ state will experience strong torque along the $\mathrm{S}-\mathrm{CH}_{3}$ torsional coordinate as the $\mathrm{S}-\mathrm{CH}_{3}$ bond is elongated. The nonplanarity of 2-fluorothioanisole in $\mathrm{S}_{2}$ would make the passage of the reactive flux remote from the $S_{0} / S_{2}$ conical intersection located on the planar geometry, providing a perfect explanation for the nearly complete absence of the $\mathrm{C}_{6} \mathrm{H}_{4} \mathrm{FS} \cdot(\tilde{\mathrm{X}})+\cdot \mathrm{CH}_{3}$ fragmentation channel.

Interestingly, in Fig. 4, the potential energy curves of the $\mathrm{S}_{1}$ and $\mathrm{S}_{2}$ state are close at the $\mathrm{S}-\mathrm{CH}_{3}$ dihedral angle of $\sim 80^{\circ}$. The conical intersection optimization shows that a quasi-degenerate $\mathrm{S}_{1} / \mathrm{S}_{2}$ surface crossing seam, where the energy gap between the lower and upper adiabats is small, exists in the nonplanar geometry, although the $\mathrm{S}_{1} / \mathrm{S}_{2}$ minimum-energy conical intersection (MECI) adopts a planar geometry, Table 1. According to our CASSCF calculations, a quasi-degenerate $S_{1} / S_{2}$ crossing seam is located $\sim 700 \mathrm{~cm}^{-1}$ below the planar MECI and $\sim 830 \mathrm{~cm}^{-1}$ above the $\mathrm{S}_{1}$ minimum. The calculated energetic position of nonplanar quasi-seam with respect to the $S_{1}$ minimum is actually very low considering the fact that CASSCF values are often overestimated. The nonplanar quasi-seam adopts a geometry where the $\mathrm{S}-\mathrm{CH}_{3}$ bond length is $2.11 \AA$ and a S- $\mathrm{CH}_{3}$ dihedral angle of $-79^{\circ}$. Since it is energetically located at a much lower position than the planar MECI, at least the reactive flux prepared in the low $S_{1}$ internal energy region may have a chance to explore the phase space around the nonplanar $\mathrm{S}_{1} / \mathrm{S}_{2}$ quasi-seam, especially since the $\mathrm{S}-\mathrm{CH}_{3}$ torsional mode is strongly activated in the $\mathrm{S}_{1}-\mathrm{S}_{0}$ optical excitation. Actually, a similar conceptual approach may underlie the previous findings that symmetry breaking in the photoexcitation of catechol, ${ }^{19,20}$ 2-aminophenol, ${ }^{22}$ or 2-fluorophenol- $\left(\mathrm{NH}_{3}\right)$ cluster $^{16}$ is responsible for the shortening of excited-state lifetimes.

It seems that strong coupling of the bound $\left(\mathrm{S}_{1}\right)$ and unbound $\left(\mathrm{S}_{2}\right)$ states in the nonplanar geometry transforms the initiallyprepared quantum state immediately into a mixture of contributions from the upper and lower adiabats. Since the quasidegenerate $S_{1} / S_{2}$ crossing seam developed in the nonplanar nuclear configuration is expected to be located just a few hundreds of $\mathrm{cm}^{-1}$ above the $\mathrm{S}_{1}$ minimum, there might be a significant chance for the reactive flux confined in the upper adiabat to leak into the repulsive $S_{2}$ state through the nonplanar quasi-seam. Since the out-of-plane $\mathrm{S}-\mathrm{CH}_{3}$ torsional mode is highly excited in $S_{1}$, the $S_{1} / S_{2}$ coupling should be very efficient over the wide range of nuclear configurations. Similar to the 
Table 1 Planar minimum-energy conical intersection (MECI) and nonplanar quasi-degenerate $\mathrm{S}_{1} / \mathrm{S}_{2}$ crossing point calculated using SA4CASSCF(12,11)/6-311++G(3df,3pd)

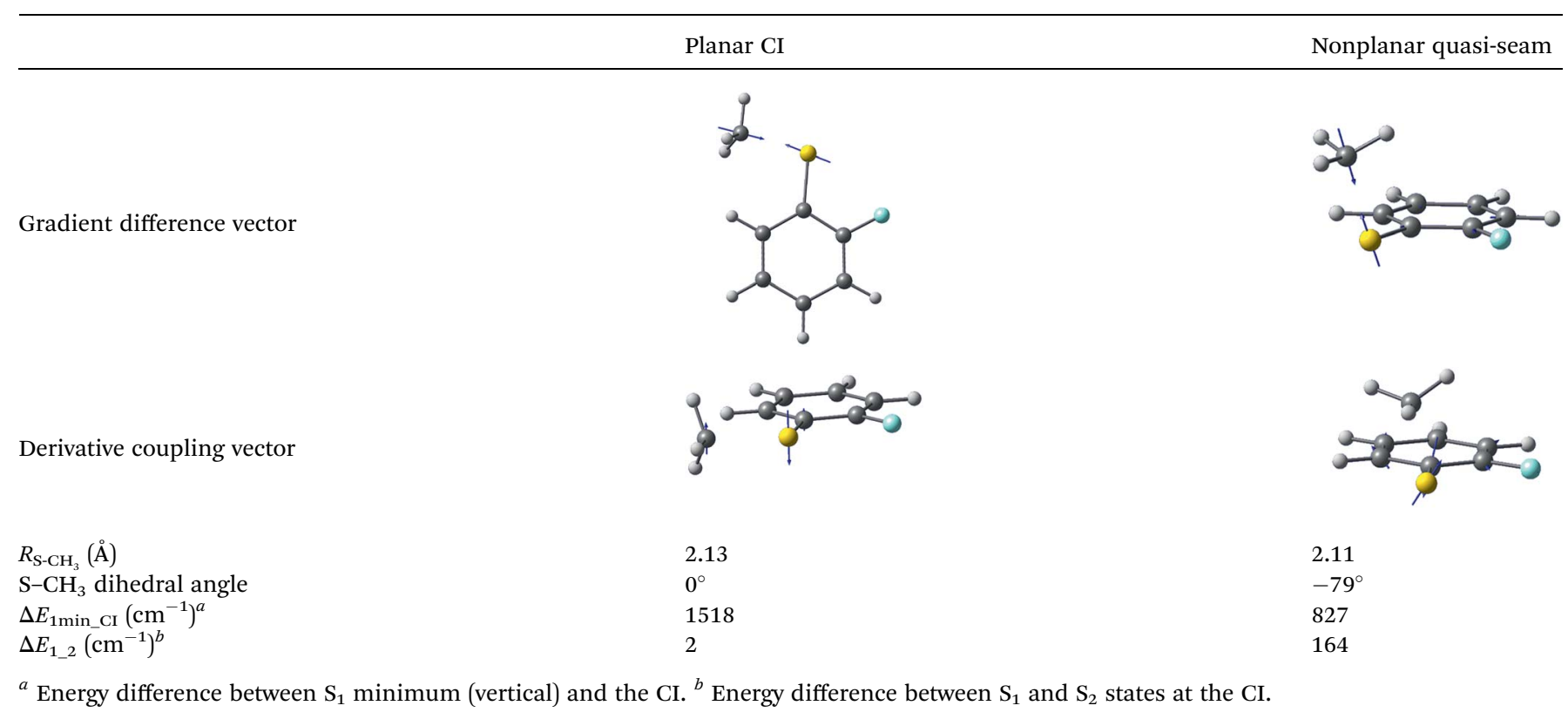

case of thioanisole, it seems straightforward to identify the origins of the high and low translational energy components in the total translational energy distributions of products from 2fluorothioanisole (vide supra). Obviously, the reactive flux initially confined in the upper adiabat gives rise to fragments less excited along the vibrational degrees of freedom orthogonal to the reaction coordinate. This should produce a larger kinetic energy release, giving the higher and narrower translational energy distribution of the fragments. Nonadiabatic leakage from the upper adiabat through either the $S_{1} / S_{2}$ conical intersection or nonplanar quasi-seam is followed by the prompt $\mathrm{S}-$ $\mathrm{CH}_{3}$ bond breakage on the repulsive $\mathrm{S}_{2}$ state (channel II). In the meantime, the relatively low component of the translational energy distribution results from exploration of the reactive flux sticking to the lower adiabatic potential energy surfaces. Here, in order to overcome the adiabatic barrier, whose top corresponds to the saddle point along the $\mathrm{S}-\mathrm{CH}_{3}$ elongation coordinate, the reactive flux explores the large volume of phase space prior to reaching the critical configurations necessary for riding on $\mathrm{S}_{2}$ (channel $\mathrm{I}$ ). Therefore, this time-consuming exploration gives rise to internally hot fragments, giving naturally low and broad translational energy product distributions. As the excitation energy increases, channel II becomes dominant with the aid of IVR, resulting in the broader translational energy distributions observed at higher excitation energies.

\section{Conclusions}

Herein, we report that the nonadiabatic bifurcation phenomenon is robust when the quantum state is prepared at resonances in the continuum generated by the strong coupling of bound and unbound states in the Franck-Condon region along the reaction coordinate. Our observation clearly demonstrates that the reactive flux in the strong bound-unbound vibronic coupling regime can be described as the quantum state of mixed characteristics belonging to either the upper or lower adiabatic states. The vibronic state mixing includes both IVR and electronic coupling beyond the Born-Oppenheimer approximation, and we determined that both Herzberg type-I (electronic) and type-II (vibrational) predissociation mechanisms coexist and proceed in a competitive way. Competition between the two mechanisms is very sensitive to the location of the reactive flux with respect to the Franck-Condon and/or coupling region in the multidimensional nuclear configuration. In 2-fluorothioanisole, it was possible to investigate the dynamics taking place in the nonplanar nuclear configurational space thanks to the particular fact that the molecule undergoes a planar-to-nonplanar structural change upon the first electronic excitation. Chemical substitution turns out to be a quite valuable tool for exploring dynamics in a variety of nuclear configurational spaces. The excitation energy dependent structures of fractions of two competing channels should represent vibronic coupling matrix elements in the expression of Fermi's golden rule. ${ }^{69}$ Full dimensional calculations of the potential energy surfaces are desirable to arrive at a quantitative explanation of the experiment in the near future.

\section{Conflicts of interest}

There are no conflicts to declare.

\section{Acknowledgements}

This work was financially supported by the Samsung Science and Technology Foundation under Project Number SSTFBA1401-09. 


\section{References}

1 U. Fano, Phys. Rev., 1961, 124, 1866-1878.

2 P. L. Altick and E. N. Moore, Phys. Rev., 1966, 147, 59-65.

3 L. S. Cederbaum, R. S. Friedman, V. M. Ryaboy and N. Moiseyev, Phys. Rev. Lett., 2003, 90, 013001.

4 C. S. Anstöter, J. N. Bull and J. R. R. Verlet, Int. Rev. Phys. Chem., 2016, 35, 509-538.

5 M. C. Bordas, L. J. Lembo and H. Helm, Phys. Rev. A: At., Mol., Opt. Phys., 1991, 44, 1817-1827.

6 J. T. Brandon, S. A. Reid, D. C. Robie and H. Reisler, J. Chem. Phys., 1992, 97, 5246-5249.

7 H. u. Suter, J. R. Huber, M. v. Dirke, A. Untch and R. Schinke, J. Chem. Phys., 1992, 96, 6727-6734.

8 S. A. Reid, J. T. Brandon and H. Reisler, J. Phys. Chem., 1993, 97, 540-543.

9 R. Cotting, J. R. Huber and V. Engel, J. Chem. Phys., 1994, 100, 1040-1048.

10 D.-S. Ahn, S.-Y. Kim, G.-I. Lim, S. Lee, Y. S. Choi and S. K. Kim, Angew. Chem., Int. Ed., 2010, 49, 1244-1247.

11 S. Matsika and P. Krause, Annu. Rev. Phys. Chem., 2011, 62, 621-643.

12 G. M. Roberts and V. G. Stavros, Chem. Sci., 2014, 5, 16981722.

13 A. L. Sobolewski and W. Domcke, J. Phys. Chem. A, 2001, 105, 9275-9283.

14 A. L. Sobolewski, W. Domcke, C. Dedonder-Lardeux and C. Jouvet, Phys. Chem. Chem. Phys., 2002, 4, 1093-1100.

15 M. N. R. Ashfold, A. L. Devine, R. N. Dixon, G. A. King, M. G. D. Nix and T. A. A. Oliver, Proc. Natl. Acad. Sci. U. S. A., 2008, 105, 12701-12706.

16 G. A. Pino, A. N. Oldani, E. Marceca, M. Fujii, S.-I. Ishiuchi, M. Miyazaki, M. Broquier, C. Dedonder and C. Jouvet, J. Chem. Phys., 2010, 133, 124313.

17 R. N. Dixon, T. A. A. Oliver and M. N. R. Ashfold, J. Chem. Phys., 2011, 134, 194303.

18 G. M. Roberts, A. S. Chatterley, J. D. Young and V. G. Stavros, J. Phys. Chem. Lett., 2012, 3, 348-352.

19 M. Weiler, M. Miyazaki, G. Féraud, S.-i. Ishiuchi, C. Dedonder, C. Jouvet and M. Fujii, J. Phys. Chem. Lett., 2013, 4, 3819-3823.

20 A. S. Chatterley, J. D. Young, D. Townsend, J. M. Zurek, M. J. Paterson, G. M. Roberts and V. G. Stavros, Phys. Chem. Chem. Phys., 2013, 15, 6879-6892.

21 S. G. Ramesh and W. Domcke, Faraday Discuss., 2013, 163, 73-94.

22 M. C. Capello, M. Broquier, S.-I. Ishiuchi, W. Y. Sohn, M. Fujii, C. Dedonder-Lardeux, C. Jouvet and G. A. Pino, J. Phys. Chem. A, 2014, 118, 2056-2062.

23 T. N. V. Karsili, A. M. Wenge, B. Marchetti and M. N. R. Ashfold, Phys. Chem. Chem. Phys., 2014, 16, 588-598.

24 K. R. Yang, X. Xu, J. Zheng and D. G. Truhlar, Chem. Sci., 2014, 5, 4661-4680.

25 C. Xie, J. Ma, X. Zhu, D. R. Yarkony, D. Xie and H. Guo, J. Am. Chem. Soc., 2016, 138, 7828-7831.
26 J. S. Lim, I. S. Lim, K.-S. Lee, D.-S. Ahn, Y. S. Lee and S. K. Kim, Angew. Chem., Int. Ed., 2006, 45, 6290-6293.

27 I. S. Lim, J. S. Lim, Y. S. Lee and S. K. Kim, J. Chem. Phys., 2007, 126, 034306.

28 A. L. Devine, M. G. D. Nix, R. N. Dixon and M. N. R. Ashfold, J. Phys. Chem. A, 2008, 112, 9563-9574.

29 J. S. Lim, H. Choi, I. S. Lim, S. B. Park, Y. S. Lee and S. K. Kim, J. Phys. Chem. A, 2009, 113, 10410-10416.

30 T. S. Venkatesan, S. G. Ramesh, Z. Lan and W. Domcke, J. Chem. Phys., 2012, 136, 174312.

31 S. Han, H. S. You, S.-Y. Kim and S. K. Kim, J. Phys. Chem. A, 2014, 118, 6940-6949.

32 H. S. You, S. Han, J. S. Lim and S. K. Kim, J. Phys. Chem. Lett., 2015, 6, 3202-3208.

33 H. S. You, S. Han, J.-H. Yoon, J. S. Lim, J. Lee, S.-Y. Kim, D.-S. Ahn, J. S. Lim and S. K. Kim, Int. Rev. Phys. Chem., 2015, 34, 429-459.

34 V. Ovejas, M. Fernández-Fernández, R. Montero and A. Longarte, Chem. Phys. Lett., 2016, 661, 206-209.

35 B. Marchetti, T. N. V. Karsili, M. Cipriani, C. S. Hansen and M. N. R. Ashfold, J. Chem. Phys., 2017, 147, 013923.

36 G.-S.-M. Lin, C. Xie and D. Xie, J. Phys. Chem. A, 2018, 122, 5375-5382.

37 J. S. Lim and S. K. Kim, Nat. Chem., 2010, 2, 627.

38 G. M. Roberts, D. J. Hadden, L. T. Bergendahl, A. M. Wenge, S. J. Harris, T. N. V. Karsili, M. N. R. Ashfold, M. J. Paterson and V. G. Stavros, Chem. Sci., 2013, 4, 993-1001.

39 S. Han, J. S. Lim, J.-H. Yoon, J. Lee, S.-Y. Kim and S. K. Kim, J. Chem. Phys., 2014, 140, 054307.

40 J. Lee, S.-Y. Kim and S. K. Kim, J. Phys. Chem. A, 2014, 118, 1850-1857.

41 A. M. Wenge, T. N. V. Karsili, J. D. Rodriguez, M. I. Cotterell, B. Marchetti, R. N. Dixon and M. N. R. Ashfold, Phys. Chem. Chem. Phys., 2015, 17, 16246-16256.

42 S.-Y. Kim, J. Lee and S. K. Kim, Phys. Chem. Chem. Phys., 2017, 19, 18902-18912.

43 S. L. Li and D. G. Truhlar, J. Chem. Phys., 2017, 147, 044311. 44 S. L. Li and D. G. Truhlar, J. Chem. Phys., 2017, 146, 064301.

45 K. C. Woo, D. H. Kang and S. K. Kim, J. Am. Chem. Soc., 2017, 139, 17152-17158.

46 Y. Shu and D. G. Truhlar, Chem. Phys., 2018, 515, 737-743. 47 C. Nicole, I. Sluimer, F. Rosca-Pruna, M. Warntjes, M. Vrakking, C. Bordas, F. Texier and F. Robicheaux, Phys. Rev. Lett., 2000, 85, 4024-4027.

48 D. M. Neumark, J. Phys. Chem. A, 2008, 112, 13287-13301.

49 W. Li, S. D. Chambreau, S. A. Lahankar and A. G. Suits, Rev. Sci. Instrum., 2005, 76, 063106.

50 V. Dribinski, A. Ossadtchi, V. A. Mandelshtam and H. Reisler, Rev. Sci. Instrum., 2002, 73, 2634-2642.

51 A. T. J. B. Eppink and D. H. Parker, Rev. Sci. Instrum., 1997, 68, 3477-3484.

52 A. E. Azhary, G. Rauhut, P. Pulay and H.-J. Werner, J. Chem. Phys., 1998, 108, 5185-5193.

53 A. D. Becke, Phys. Rev. A: At., Mol., Opt. Phys., 1988, 38, 30983100.

54 F. Weinhold, J. Comput. Chem., 2012, 33, 2363-2379. 
55 T. Korona and H.-J. Werner, J. Chem. Phys., 2003, 118, 30063019.

56 P. J. Knowles and H.-J. Werner, Chem. Phys. Lett., 1985, 115, 259-267.

57 H. J. Werner and P. J. Knowles, J. Chem. Phys., 1985, 82, 5053-5063.

58 M. J. Frisch, G. W. Trucks, H. B. Schlegel, G. E. Scuseria, M. A. Robb, J. R. Cheeseman, G. Scalmani, V. Barone, B. Mennucci, G. A. Petersson, H. Nakatsuji, M. Caricato, X. Li, H. P. Hratchian, A. F. Izmaylov, J. Bloino, G. Zheng, J. L. Sonnenberg, M. Hada, M. Ehara, K. Toyota, R. Fukuda, J. Hasegawa, M. Ishida, T. Nakajima, Y. Honda, O. Kitao, H. Nakai, T. Vreven, J. A. Montgomery, J. E. Peralta, F. Ogliaro, M. Bearpark, J. J. Heyd, E. Brothers, K. N. Kudin, V. N. Staroverov, R. Kobayashi, J. Normand, K. Raghavachari, A. Rendell, J. C. Burant, S. S. Iyengar, J. Tomasi, M. Cossi, N. Rega, J. M. Millam, M. Klene, J. E. Knox, J. B. Cross, V. Bakken, C. Adamo, J. Jaramillo, R. Gomperts, R. E. Stratmann, O. Yazyev, A. J. Austin, R. Cammi, C. Pomelli, J. W. Ochterski, R. L. Martin, K. Morokuma, V. G. Zakrzewski, G. A. Voth, P. Salvador, J. J. Dannenberg, S. Dapprich, A. D. Daniels, O. Farkas, J. B. Foresman, J. V. Ortiz, J. Cioslowski and D. J. Fox, Gaussian 09 (Revision D.01), Gaussian Inc., Wallingford, CT, 2009.

59 H.-J. Werner, P. J. Knowles, G. Knizia, F. R. Manby, M. Schutz, P. Celani, T. Korona, R. Lindh, A. Mitrushenkov, G. Rauhut, K. R. Shamasundar, T. B. Adler, R. D. Amos, A. Bernhardsson, A. Berning,
D. L. Cooper, M. J. O. Deegan, A. J. Dobbyn, F. Eckert, E. Goll, C. Hampel, A. Hesselmann, G. Hetzer, T. Hrenar, G. Jansen, C. Koppl, Y. Liu, A. W. Lloyd, R. A. Mata, A. J. May, S. J. McNicholas, W. Meyer, M. E. Mura, A. Nicklass, D. P. O'Neill, P. Palmieri, K. Pfluger, R. Pitzer, M. Reiher, T. Shiozaki, H. Stoll, A. J. Stone, R. Tarroni, T. Thorsteinsson, M. Wang and A. Wolf, MOLPRO version 2010.1, a package of ab initio programs, see http:// www.molpro.net.

60 D. Chmielewski, N. H. Werstiuk and T. A. Wildman, Can. J. Chem., 1993, 71, 1741-1750.

61 T. Vondrák, S.-i. Sato, V. Špirko and K. Kimura, J. Phys. Chem. A, 1997, 101, 8631-8638.

62 M. Nagasaka-Hoshino, T. Isozaki, T. Suzuki, T. Ichimura and S. Kawauchi, Chem. Phys. Lett., 2008, 457, 58-61.

63 I. F. Shishkov, L. V. Khristenko, N. M. Karasev, L. V. Vilkov and H. Oberhammer, J. Mol. Struct., 2008, 873, 137-141.

64 M. Hoshino-Nagasaka, T. Suzuki, T. Ichimura, S. Kasahara, M. Baba and S. Kawauchi, Phys. Chem. Chem. Phys., 2010, 12, 13243-13247.

65 M. Gerhards, S. Schumm, C. Unterberg and K. Kleinermanns, Chem. Phys. Lett., 1998, 294, 65-70.

66 L. Yuan, C. Li, J. L. Lin, S. C. Yang and W. B. Tzeng, Chem. Phys., 2006, 323, 429-438.

67 K. L. Reid, Int. Rev. Phys. Chem., 2008, 27, 607-628.

68 J. B. Kim, T. I. Yacovitch, C. Hock and D. M. Neumark, Phys. Chem. Chem. Phys., 2011, 13, 17378-17383.

69 P. Avouris, W. M. Gelbart and M. A. El-Sayed, Chem. Rev., 1977, 77, 793-833. 